\title{
Carbon Nanotubes in a Photonic Metamaterial
}

\author{
Andrey E. Nikolaenko, ${ }^{1}$ Francesco De Angelis, ${ }^{2}$ Stuart A. Boden, ${ }^{3}$ Nikitas Papasimakis, ${ }^{1}$ Peter Ashburn, ${ }^{3}$ \\ Enzo Di Fabrizio, ${ }^{2}$ and Nikolay I. Zheludev ${ }^{1}$ \\ ${ }^{1}$ Optoelectronics Research Centre, University of Southampton, Southampton SO17 1BJ, United Kingdom \\ ${ }^{2}$ Italian Institute of Technology, 16163 Genova and the University of Magna Graecia, 88100 Catanzaro, Italy \\ ${ }^{3}$ School of Electronics and Computer Science, University of Southampton, Southampton SO17 1BJ, United Kingdom
}

(Received 11 December 2009; revised manuscript received 9 March 2010; published 12 April 2010)

Hybridization of single-walled carbon nanotubes with plasmonic metamaterials leads to photonic media with an exceptionally strong ultrafast nonlinearity. This behavior is underpinned by strong coupling of the nanotube excitonic response to the weakly radiating Fano-type resonant plasmonic modes that can be tailored by metamaterial design.

DOI: 10.1103/PhysRevLett.104.153902

Metamaterials, artificial media structured on the subwavelength scale, offer new photonic functionalities ranging from negative index of refraction and directionally asymmetric transmission to slowing light. Owing to the resonant nature of the metamaterial response which leads to strong light localization, combinations of metamaterials with nonlinear media are expected to present giant nonlinearities [1] enabling optical tuning and switching of the metamaterial properties. However, despite the growing body of theoretical investigations on nonlinear metamaterials [2-6] and the tremendous application potential, most experimental work takes place in the microwave domain [7-10] with optical experiments being limited to studies of higherharmonic generation [11-13]. Here we demonstrate experimentally a new paradigm for the development of media with enhanced nonlinear performance: A combination of carbon nanotubes (CNTs) with a photonic metamaterial, where the strong coupling between "dark", weakly radiating plasmonic modes and the excitonic response of singlewalled semiconductor CNTs leads to exceptionally strong and ultrafast nonlinear tuning of transmission.

Carbon nanotubes are nearly ideal one-dimensional systems, with diameter of a few nanometers and length on the micron scale. Single-walled CNTs rolled from a graphene sheet are direct gap semiconductors with absorption spectra dominated by exciton lines [14]. Possible technological uses include nanoscale light sources, photodetectors, and photovoltaic devices. CNTs possess unique nonlinear optical properties [15] as they exhibit high third-order susceptibility with subpicosecond recovery time [16,17] lending to applications in ultrafast lasers [18-22]. CNTs are very appealing nonlinear media due to simple and cheap fabrication methods, robustness, and easy integration into waveguide environments. The main source of nonlinearity in semiconductor CNTs is the saturation of the resonant exciton line. Combining CNTs with metamaterials allows us to engage the resonant local fields in the vicinity of the metamaterial leading to enhanced nonlinearity. We used a planar metamaterial that supports darkmode excitations [23], where weak coupling of the excited
PACS numbers: 42.70.Mp, 78.67.Pt

plasmonic mode to free-space radiation modes creates narrow Fano-like resonances. The first example of such a metamaterial was a periodic array of metallic asymmetrically split ring (ASR) wire resonators that has found numerous applications [24-27]. Here we used a complementary structure: a periodic array of ASR slits in a metal film (see Fig. 1).

In our experiments, we followed a combinatorial approach, where a rapid search for the optimal composition is achieved by parallel screening of a number of structurally related samples [28]. The unit cell size, $D$, of the studied metamaterial arrays varied from 731 to $839 \mathrm{~nm}$ (see supplementary material [29]). Since the position of the plasmonic resonance $\lambda_{p}$ depends on $D$, we were able to study the dependence of the response on the spectral separation, $\delta_{\mathrm{pe}}=\lambda_{11}-\lambda_{p}$, of the main CNT excitonic resonance $\left(\lambda_{11}\right)$ from the metamaterial plasmonic resonance $\left(\lambda_{p}\right)$. Characteristic spectra of the metamaterial are presented on Fig. 2(a) for an array with $D=731 \mathrm{~nm}$. Figure 3(d) shows the dependence of the peak of the metamaterial plasmon absorption line, $\lambda_{p}$, on $D$.

The metamaterials were functionalized by depositing a thin layer of single-walled CNTs [29,30]. Figure 1 shows images of the metamaterial before and after functionalization with CNTs, taken with a scanning helium ion microscope, where the CNTs seem to form a strongly interlinked network, while single CNTs bridging the gaps of the metal nanostructure are also seen [see Fig. 1(e)]. We investigated the morphology of the CNT metamaterial by observing its cross section in a trench cut through the sample by a focused ion beam [Fig. 1(f)]. For this matter, a section of the sample was covered by a thin protective layer of tungsten. The layer of CNTs had a thickness between 20 and $70 \mathrm{~nm}$ across the sample [Fig. 1(g)] and creates negligible scattering due to its deep subwavelength structure. The CNT layer shows a characteristic absorption spectrum dominated by $\lambda_{11}$ and $\lambda_{22}$ excitonic lines, as presented in Fig. 3(b).

We observed substantial changes in the metamaterial's optical properties resulting from the CNT functional- 


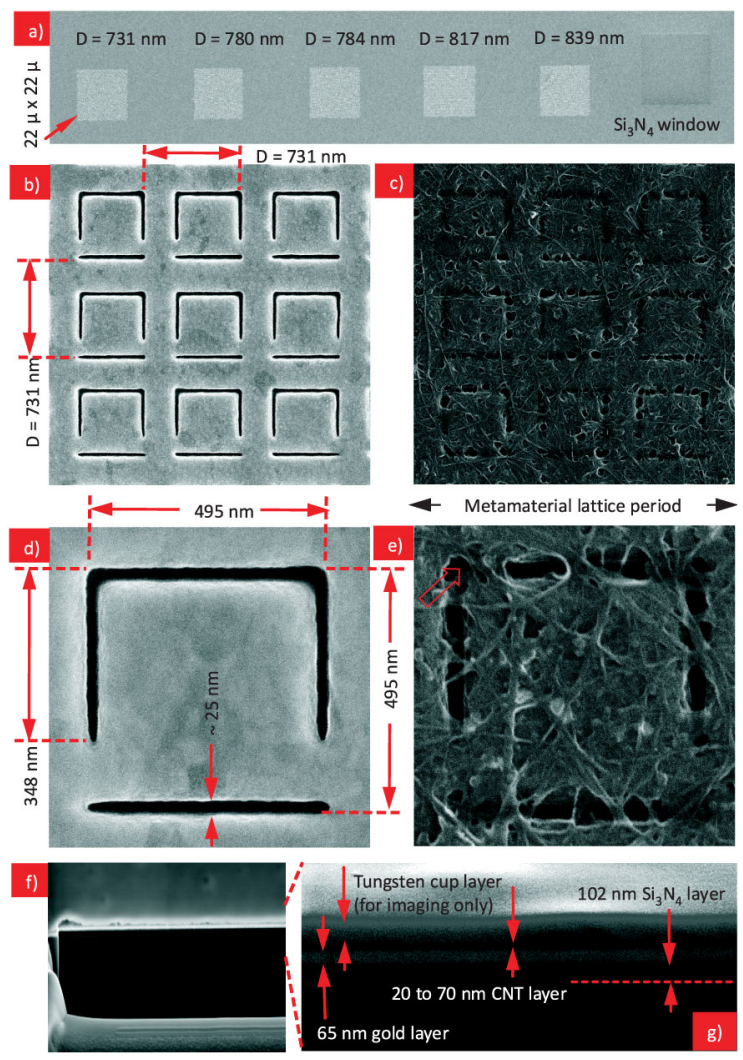

FIG. 1 (color online). Carbon nanotube metamaterial imaged under a scanning helium ion microscope. (a) The combinatorial sample consists of five metamaterial arrays with different unit cell sizes $D$ and an empty area annotated as " $\mathrm{Si}_{3} \mathrm{~N}_{4}$ window" on the same substrate. (b) The metamaterial structure is an array of slits in a gold film supported on a $\mathrm{Si}_{3} \mathrm{~N}_{4}$ membrane. (c) CNTs deposited on the surface of the metal nanostructure form a layer of "nanoscale feutre." Plates (d) and (e) show unit cells of the metamaterial before and after deposition of nanotubes. On plate (e) note the arrow pointing at a single nanotube crossing the slit. Plate (f) shows a milled slit manufactured to study the morphology of the structure, which is presented in plate $(\mathrm{g})$.

ization. All resonance features exhibited an anticipated "redshift" $\Delta=\lambda_{p}^{*}-\lambda_{p}$ of the plasmon resonance resulting from the reduction of the plasmon frequency due to the presence of the highly polarizable CNTs [compare Figs. 2(a) and 2(b)]. After functionalization the metamaterial's reflection decreased, whereas the absorption spectrum revealed a background associated with the nanotube interband transitions and increased damping linked to the metallic component of the CNT mixture. Additional losses introduced by the CNTs damped the metamaterial plasmonic resonance; hence its quality factor decreased. Hidden in the stronger spectral features of the metal nanostructure, the $\lambda_{11}$ excitonic line is not identifiable on the absorption spectrum of the functionalized metamaterial. The redshifted positions of the trapped mode resonance in the metamaterial-CNT system are presented in Fig. 3(d) for different values of $D$.

The nonlinear response of the metamaterial was investigated with a broadband supercontinuum source with a

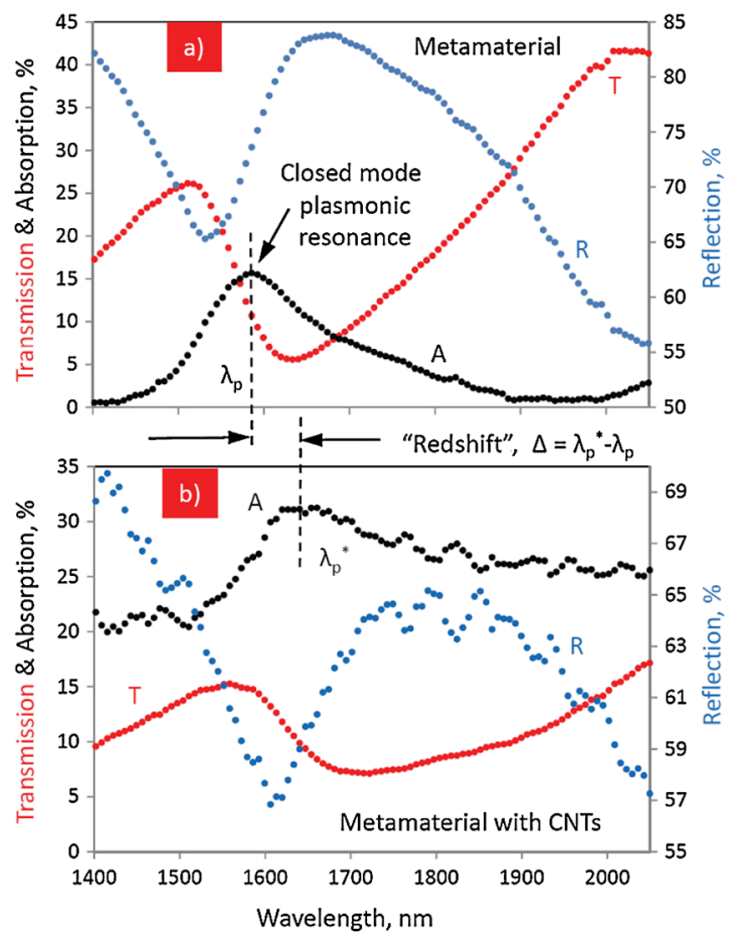

FIG. 2 (color online). Spectral response of the carbon nanotube metamaterial: transmission $(T)$, reflection $(R)$ and absorption $(A)$ for a metamaterial array with a $D=731 \mathrm{~nm}$ unit cell size (a) and for the same metamaterial fuctionalized with CNTs (b). Note the redshift of the plasmon absorption resonance in the CNT-fuctionalized metamaterial.

repetition rate of $20 \mathrm{MHz}$ (see supplementary material [29]). Since the pulse duration was a function of wavelength, we present the results in terms of fluence of the light excitation. The spectra of the nonlinear response are presented on Fig. 4. Here the nonlinear response is normalized to the fluence level of $40 \mu \mathrm{J} / \mathrm{cm}^{2}$ across the entire spectrum. At resonance, the light-induced transmitted intensity variation of the CNT metamaterial is about $10 \%$. In good agreement with previous works [21,22], we detected a much weaker nonlinear response of CNTs on the unstructured dielectric substrate [Fig. 4(a)] from which we were able to confirm that the metallic CNTs do not modify significantly the, mainly excitonic, response of the CNT layer. This is also true for any proximity effects due to the bundling of the CNTs [31].

The nonlinear response of the metamaterial has a complex frequency dispersion that can be decomposed on two main components: The first is practically independent of the unit cell size and is relevant to the bleaching of the CNT exciton resonance. Here increase of the light intensity leads to transmission increase. On Fig. 4(b) this is illustrated by a dashed bell-shaped line with amplitude $A_{1}$ that is centered at the CNT's exciton absorption peak at $1950 \mathrm{~nm}$ and has a width of about $310 \mathrm{~nm}$. This bleaching response is superimposed to a much sharper "negative" peak of reduced transmission. For a metamaterial with $D=839 \mathrm{~nm}$ this peak, indicated as $A_{2}$, has a width of about $120 \mathrm{~nm}$. We 

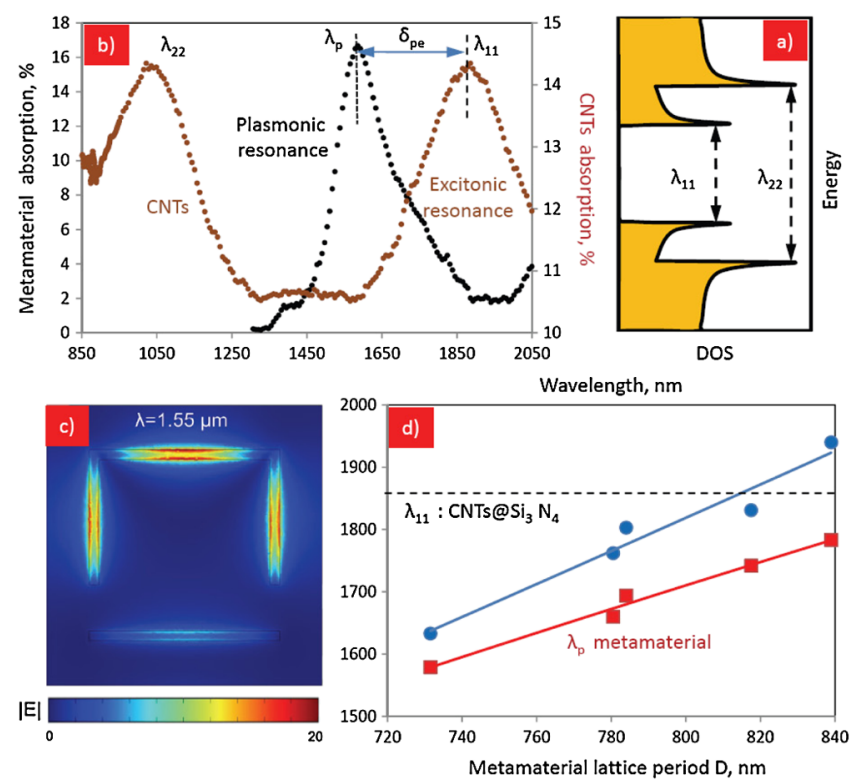

FIG. 3 (color online). Electronic density of states (DOS) in a semiconductor single-walled CNT (a); plasmonic absorption resonance in a metamaterial without CNTs and excitonic resonances in a CNT film on a silicon nitride substrate (b); simulated (COMSOL 3.4) color coded field map showing the total magnitude of the electric field of the light wave in the immediate proximity of the metamaterial plane at the plasmonic resonance $\lambda_{p}$ (c); and the dependence of the metamaterial absorption resonance spectral position on the unit cell size before and after functionalization with CNTs (d).

argue that this component is linked to the reduced damping of the plasmon mode through exciton-plasmon coupling. Under strong resonant coupling, the lower exciton damping results in the plasmon absorption peak becoming more intense and partially recovering the low transmission levels characteristic of the "CNT-free" metamaterial. This inter-

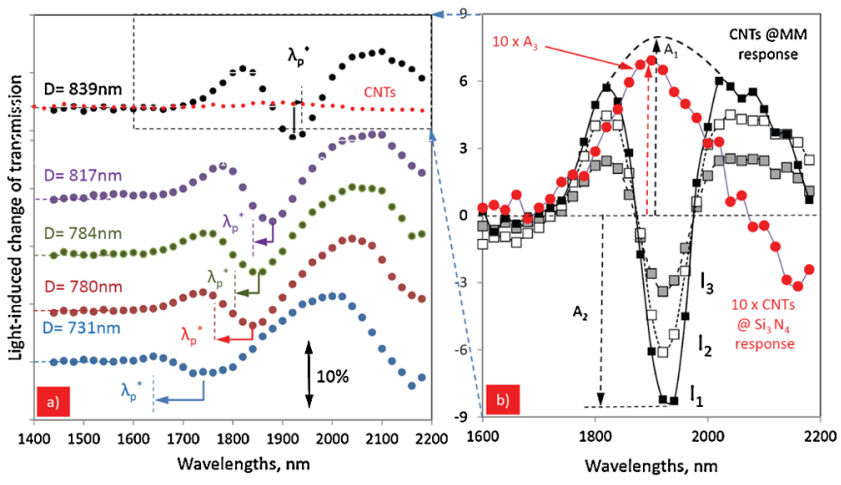

FIG. 4 (color online). Light-induced change of transmission of the CNT-functionalized metamaterial for different unit cell sizes (a). Light-induced transmission change for different levels of intensity for a CNT-functionalized metamaterial with unit cell size $D=839 \mathrm{~nm}$ (b). The nonlinear response of CNTs on a silicon nitride membrane (magnified by a factor of 100) is shown for comparison on panel (b) and also on panel (a) (without magnification). pretation is very well supported by the fact that the negative peak blue shifts with decreasing unit cell size (decreasing resonant wavelength).

The nonlinear effect here has a transient nature, and involves nonlinear refraction, leading to dynamic resonance pulling, where the apparent resonance frequency of the negative response lies in between the exciton line and the plasmon resonance. When the plasmon and exciton resonances nearly coincide [see Fig. 4(b)], the negative effect is most pronounced. This nonlinear response may be compared with that of CNTs deposited on a bare $\mathrm{Si}_{3} \mathrm{~N}_{4}$ membrane: the CNT's response on the unstructured substrate (peak $A_{3}$ ) is about 12 times smaller than the overall negative response of the CNTs on the metamaterial (peak $A_{2}$ ). Also, the positive response of the CNTs (peak $A_{3}$ ) is a factor of 13 smaller than that of the positive component of the CNT's response on the metamaterial (peak $A_{1}$ ), while the overall negative response (peak $A_{1}$ plus peak $A_{2}$ ) is a factor of 25 smaller than that of the CNTs alone. This enhanced nonlinear response is due to the strong resonant plasmon fields in the vicinity of the ASR slits [see Fig. 3(c)] at the plasmon resonance $\left(\lambda_{p}\right)$.

The main features of the mechanism underlying the nonlinear optical response of the CNT metamaterial can be understood in terms of a simple mechanical model consisting of coupled Hooke oscillators (mass on elastic spring) driven by an external harmonic force $F$ [see Fig. 5(a), a detailed description of the model is presented in the supplementary material] [32]. Here the plasmon resonance is represented by the oscillation of two masses, $M_{1}$ and $M_{2}$, coupled through a third mass, $M_{3}$. The oscillators represent excitations in the $\Pi$-shaped and straight slit of the ASR, while friction $\Gamma$ stands for the plasmonic losses. The mass $M_{3}$ linking the two oscillators is also damped to account for the radiation losses $\gamma_{r}$. At the dark-mode resonance the larger masses oscillate with opposite phases leaving the middle mass still. Hence radiation losses are at minimum and all the energy is stored in the oscillations of masses $M_{1}$ and $M_{2}$ leading to a sharp absorption peak associated with friction $\Gamma$ as seen in the corresponding dissipation spectrum of Fig. 5(b). To account for the resonant excitonic response of the CNT layer we introduce two nonlinear oscillators with masses $M_{4}$ and $M_{5}$, subject to nonlinear dissipation $(\beta)$ reproducing the saturation of the excitonic absorption in the CNTs. The plasmon-exciton coupling is represented by springs of constant $K_{c}$. The model reproduces all the essential features observed in the nonlinear response of the CNT metamaterial: When measured separately, the plasmon resonance appears at a frequency slightly higher then the CNT excitonic resonance and is sharper [see Fig. 5(b)]. For small amplitude of the driving force $F$ (low light intensity), the dark-mode experiences strong damping due to plasmon-exciton coupling. For high level of excitation (high light intensity), the excitonic absorption saturates and hence the plasmonic peak, now subject to lower losses, partially recovers, increasing in 

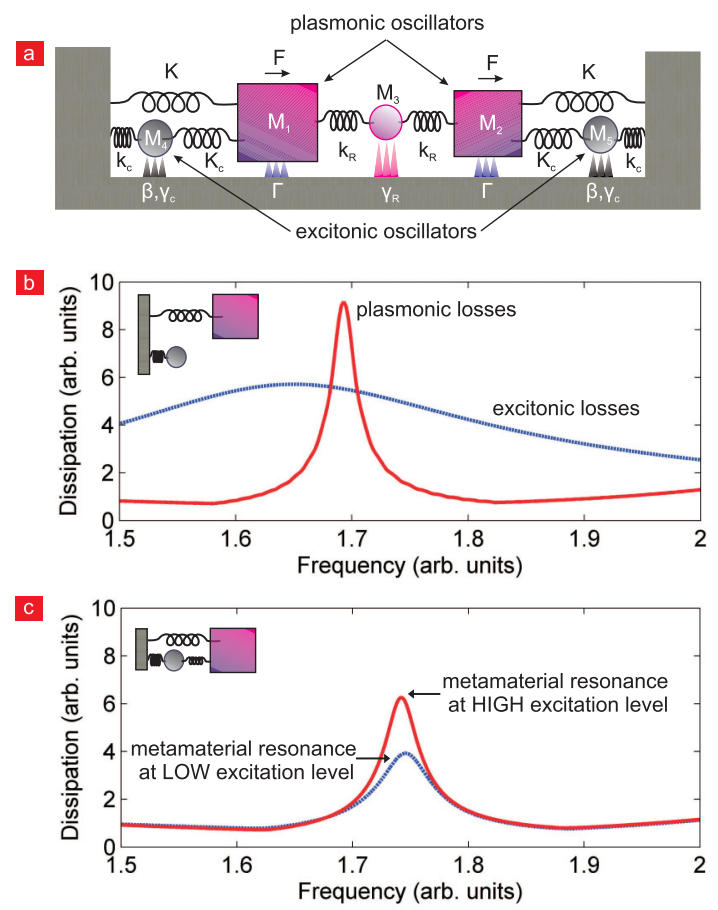

FIG. 5 (color online). (a) Illustrative model of the CNT metamaterial and the plasmon-exciton nonlinearity. (See text and supplementary material [29] for more details.) (b) Linear dissipation of the uncoupled plasmon (red line) and exciton (blue line) systems. (c) Dissipation losses of the linked plasmonexciton system at different levels of excitation.

amplitude and becoming narrower [see Fig. 5(b)]. This illustrates our experimental observation that in the strong exciton-plasmon coupling regime (small $\delta_{\text {pe }}$ ) the transmission through a metamaterial sample becomes lower at higher levels of excitation in spite of bleaching of the exciton absorption [compare with Fig. 4(b)].

In summary we have demonstrated that CNTs can be used as very efficient nonlinearity agents for metamaterials, where exciton-plasmon coupling results in a nonlinear response at least an order of magnitude stronger than that of a bare CNT film. As the exciton-plasmon coupling provides an additional relaxation mechanism, we expect the response of the CNT metamaterial to be at least as fast as that of a CNT film (<600 fs) [17]. Also, the metamaterial environment allows us to spectrally tailor the nonlinear response and even reverse the sign of nonlinearity. We argue that CNTs on metamaterials can offer performance that is robust, stable and free from permanent bleaching. The resonant nonlinear properties of the CNT metamaterial can be easily tuned throughout the near-IR by employing CNTs of different diameter and appropriately scaling the metamaterial. At optical frequencies Joule losses in metals are unavoidable and either design optimization or loss compensation by a gain medium will be needed [24,33]. In fact, the nonlinearity of the CNT metamaterial itself may be used in parametric loss-compensation schemes. This makes CNT metamaterials promising media for ap- plications that demand thin but highly-nonlinear layers and can tolerate or require some level of losses. This includes optical limiting and all-optical switching, mode-locking and $Q$ switching of low-power lasers, as well as more exotic applications such as the time reversal negative refraction device [34].

The authors would like to acknowledge the financial support of the Engineering and Physical Sciences Research Council (U.K.) and the Royal Society.

[1] J. B. Pendry et al., IEEE Trans. Microwave Theory Tech. 47, 2075 (1999).

[2] A. A. Zharov, I. V. Shadrivov, and Y. S. Kivshar, Phys. Rev. Lett. 91, 037401 (2003).

[3] M. Gorkunov and M. Lapine Phys. Rev. B 70, 235109 (2004).

[4] S. O'Brien et al., Phys. Rev. B 69, 241101(R) (2004).

[5] A. K. Popov and V.M. Shalaev, Appl. Phys. B 84, 131 (2006).

[6] S. Feng and K. Halterman Phys. Rev. Lett. 100, 063901 (2008).

[7] I. Gil et al., Electron. Lett. 40, 1347 (2004).

[8] I. V. Shadrivov, S. K. Morrison, and Y.S. Kivshar, Opt. Express 14, 9344 (2006).

[9] A. Degiron, J. J. Mock, and D. R. Smith, Opt. Express 15, 1115 (2007).

[10] D. A. Powell, I. V. Shadrivov, and Y.S. Kivshar, Appl. Phys. Lett. 95, 084102 (2009).

[11] M. W. Klein et al., Science 313, 502 (2006).

[12] F. B. P. Niesler et al., Opt. Lett. 34, 1997 (2009).

[13] E. Kim et al., Phys. Rev. B 78, 113102 (2008).

[14] Ph. Avouris, J. Chen, M. Freitag, V. Perebeinos, and J. C. Tsang, Phys. Status Solidi B 243, 3197 (2006).

[15] P. Avouris, M. Freitag, and V. Perebeinos, Nat. Photon. 2, 341 (2008).

[16] Y.-C. Chen et al., Appl. Phys. Lett. 81, 975 (2002).

[17] S. Tatsuura et al., Adv. Mater. 15, 534 (2003).

[18] S. Y. Set et al., J. Lightwave Technol. 22, 51 (2004).

[19] J. W. Nicholson et al., Opt. Express 15, 9176 (2007).

[20] T. R. Schibli et al., Opt. Express 13, 8025 (2005).

[21] K. H. Fong et al., Opt. Lett. 32, 38 (2007).

[22] J. H. Yim et al., Appl. Phys. Lett. 93, 161106 (2008).

[23] V. A. Fedotov et al., Phys. Rev. Lett. 99, 147401 (2007).

[24] E. Plum et al., Opt. Express 17, 8548 (2009).

[25] M. J. Dicken et al., Opt. Express 17, 18330 (2009).

[26] E. Plum et al., Phys. Rev. Lett. 102, 113902 (2009).

[27] N. P. Johnson et al., Proc. SPIE Int. Soc. Opt. Eng. 6987, $69871 \mathrm{~F}$ (2008).

[28] X.-D. Xiang et al., Science 268, 1738 (1995).

[29] See supplementary material at http://link.aps.org/ supplemental/10.1103/PhysRevLett.104.153902 for details.

[30] J. W. G. Wildöer et al., Nature (London) 391, 59 (1998).

[31] M. Zamkov et al., Chem. Phys. Lett. 437, 104 (2007).

[32] This model was originally suggested by V. Fedotov (unpublished); see N. Papasimakis and N. I. Zheludev Opt. Photonics News 20, 22 (2009).

[33] N. I. Zheludev et al., Nat. Photon. 2, 351 (2008).

[34] J. B. Pendry, Science 322, 71 (2008). 